\title{
Analysis of Atomic Oxygen Fluence Distribution on Satellite Surface*
}

\author{
By Minoru IwATA, ${ }^{1)}$ Toshiaki OGAwA, ${ }^{2)}$ Hiroaki KoBAYASHI, ${ }^{3)}$ Mengu CHO, ${ }^{1)}$ \\ Jeong-ho KIM, ${ }^{4)}$ Shinji HATTA ${ }^{4)}$ and Shoichiro MiHARA ${ }^{5)}$ \\ ${ }^{1)}$ Kyushu Institute of Technology, Kitakyushu, Japan \\ ${ }^{2)}$ NEC Corporation, Tokyo, Japan \\ ${ }^{3)}$ I-NET Corporation, Yokohama, Japan \\ ${ }^{4)}$ MUSCAT Space Engineering Co. Ltd., Kitakyushu, Japan \\ 5) Japan Space Systems, Tokyo, Japan
}

(Received November 15th, 2011)

\begin{abstract}
The atomic oxygen $(\mathrm{AO})$ in low Earth orbit erodes spacecraft materials. Since the organic materials are significantly damaged by AO, we must evaluate the erosion effects during satellite missions before launch. The first step in evaluating the erosion effect due to AO is to determine the distribution of AO fluence and/or flux on the entire satellite surface during the satellite mission. The AO fluence on the satellite surface is different for different parts of the satellite surface because the ram surface receives AO changes according to the satellite attitude in orbit. Therefore, AO fluence must be estimated by considering the satellite attitude, shape, ram direction and AO density in orbit. However, there are no studies in which the AO fluence distribution is estimated for the entire satellite surface. In addition, there is no tool to analyze AO fluence distribution on the satellite surface. Thus, in this study, we construct an AO fluence distribution analysis tool and estimate the AO fluence distribution on the entire satellite surface.
\end{abstract}

Key Words: Atomic Oxygen, Surface Distribution, Fluence, Flux

\section{Introduction}

Materials used for spacecraft are exposed to harsh space environments, and are degraded by various environmental factors, such as charged particle radiation, ultraviolet rays, atomic oxygen (AO), X-rays, the thermal cycle and vacuum. The relative impact of space environments in degrading materials depends on the orbit in which the spacecraft is in. For instance, surface erosion due to AO is dominant in low Earth orbit (LEO). The erosion of polymer materials due to $\mathrm{AO}$ is a serious problem. It is estimated that the polyimide thickness erodes by several tens of micrometers due to AO exposure of the International Space Station (ISS) every year. ${ }^{1)}$ Almost all polymer-base thermal control materials and insulation materials are eroded by $\mathrm{AO}$ exposure. It is necessary to estimate the AO fluence, defined as the number of particles incident on a unit area, in this case the satellite surface, and to take measures such as altering the surface materials or increasing the thickness of polymer materials, at the beginning of satellite project.

On the other hand, the space environment exposure experiments in LEO have been performed on satellites, ${ }^{2)}$ space shuttles, ${ }^{3)}$ and the ISS $^{4,5)}$ for the purpose of assessing reaction efficiency, which is defined as the volume of materials removed by $\mathrm{AO}$ atoms, on various materials. Experiments have been also performed for the verification test of AO-resistant materials in orbit, and to clarify the

(C) 2012 The Japan Society for Aeronautical and Space Sciences *Presented at the 28th International Symposium on Space Technology and Science, June 5- 12, 2011, Okinawa, Japan erosion mechanism. In the experiment, we must quantify $\mathrm{AO}$ fluence, that is, the total number of incident $\mathrm{AO}$ atoms. To evaluate $\mathrm{AO}$ resistance of materials, the fluence of $\mathrm{AO}$ incident to the samples must be determined. After finishing the exposure test, AO fluence can be estimated using the measurement results of mass loss on monitor materials before and after exposure. However, for the adequate exposure experiment, it is necessary to predict AO fluence and then plan sample thickness and positioning.

The AO fluence, as mentioned above, is generally estimated using a neutral atmospheric model. However, up until now, the AO fluence has only been roughly estimated. There is no analysis result of $\mathrm{AO}$ fluence distribution covering the whole external surface of a satellite. The purpose of this work is to develop an AO fluence analysis tool covering the whole external surface of a spacecraft and to estimate the $\mathrm{AO}$ fluence distribution on a satellite.

\section{Neutral Atmospheric Model}

NRLMSISE-00 is an empirical neutral atmospheric model based on ground-, rocket- and satellite-based measurements. ${ }^{6} \quad$ The model provides composition, density and temperature. Input parameters are day of year, universal time, altitude, geodetic latitude and longitude, local time, 81-day average of F10.7 flux, daily F10.7 flux for the previous day and the daily magnetic index Ap. F10.7 flux is defined as a measure of the intensity of solar radio emission at $10.7 \mathrm{~cm}$ in wavelength. F10.7 flux indicates 
solar activity. The Ap index is defined as the average of eight indexes derived from three-hourly measurement values of geomagnetic disturbance. The Ap index indicates geomagnetic activity. In this study, we estimate the AO density around a satellite using the NRLMSISE-00 model.

\subsection{AO density distribution around Earth}

(a) Local time dependence

Figure 1 shows the AO density distribution at an altitude of $504 \mathrm{~km}$ and 00:00UT (universal time). The AO density reaches a maximum on the dayside and varies with universal time.

(b) Dependence on day of year

Figure 2 shows the dependence of AO density on the day of year at a longitude of 0 degrees, an altitude of $504 \mathrm{~km}$ and 00:00UT. The AO density reaches a maximum in November and minimum in July. The AO density in November is double that in July at the equator.

(c) Dependence on F10.7 flux and Ap index

Figure 3 shows the dependence of AO density on F10.7 flux and Ap index at a latitude of 0 degrees and 00:00UT in January. The AO density increases with solar and geomagnetic activity, since F10.7 flux and Ap index indicate solar and geomagnetic activity, respectively.

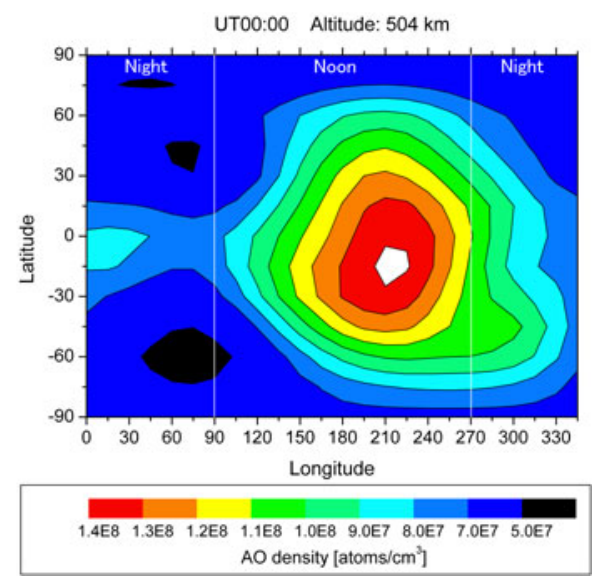

Fig. 1. Local time dependence of $\mathrm{AO}$ density.

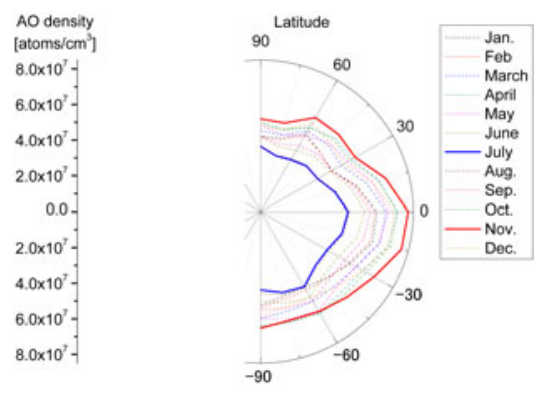

Fig. 2. Dependence of AO density on a day of year.

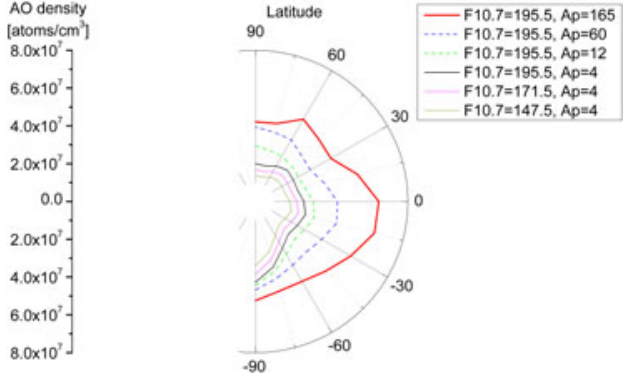

Fig. 3. Dependence of AO density on F10.7 flux and Ap index.

\section{Satellite Modeling and Simulation}

\subsection{Spacecraft ASNARO}

The Advanced Satellite with New system Architecture for Observation (ASNARO), is planned for launch in 2012 on a sun synchronous sub-recurrent orbit with an altitude of $504 \mathrm{~km}$ and an inclination of 97.4 degrees. ASNARO has open architecture technologies enabling development of a small next-generation satellite at low cost and in a short development period. ${ }^{7}$ The 400 kg-class small Earth-observation satellite has an expected ground sample distance of less than $0.5 \mathrm{~m}$ and is rated on par with large high-performance satellites worldwide. Since the atmosphere at an altitude of $504 \mathrm{~km}$ includes AO, erosion due to $\mathrm{AO}$ is a serious problem. Therefore, in this study, we analyze AO fluence distribution on the ASNARO satellite surface for a three-year design life.

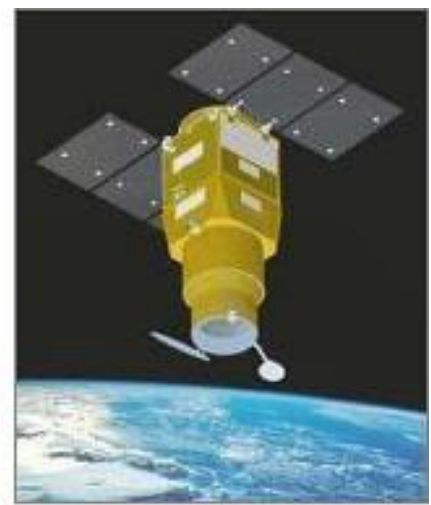

Fig. 4. ASNARO.

\subsection{Satellite modeling}

At the present time, there is no software for AO flux distribution analysis of the whole surface of spacecraft in Japan. In this study, we utilized the function of the multi-utility spacecraft charging analysis tool (MUSCAT) ${ }^{8)}$ that is used for spacecraft charging analysis. In MUSCAT, a 3D satellite model can be constructed simply via "building blocks" using a graphical user interface on a personal computer as shown in Fig. 5.

In order to achieve the original goal of spacecraft charging analysis, MUSCAT equips the solver to track charged particles, that is, electrons and ions. Unlike the motion of a charged particle in spacecraft charging analysis, AO shows linear motion relative to spacecraft. Therefore, we modified the configuration of the particle tracking solver 
in MUSCAT to the configuration under which no charged particle is affected by the electrical field, and then utilized MUSCAT to analyze the AO fluence distribution on the spacecraft surface.

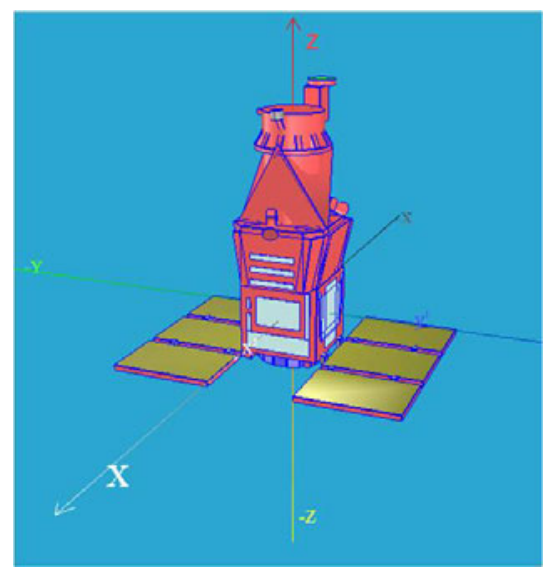

Fig. 5. 3D satellite model constructed using MUSCAT. The $-\mathrm{Z}$ axis is the direction of the sun.

\subsection{Analysis methods}

In this analysis, local times at the ascending and descending nodes on the planned ASNARO orbit described in section 3.1 are assumed to be 23:15 and 11:15, respectively, as shown in Fig. 6. We constructed a digital 3D model of the ASNARO satellite and estimated AO flux distribution on the satellite surface taking into account $\mathrm{AO}$ density at an orbital position and the relationship between satellite attitude and ram direction in orbit. The ram direction refers to the way that the spacecraft is traveling. It is assumed that the satellite passed through the $\mathrm{AO}$ atmosphere with thermal velocity and AO moves around behind the satellite wake-side surface with thermal velocity. The wake surface means the opposite side to the surface facing the direction of the spacecraft velocity vector. To estimate the thermal velocity of AO, we assume that temperature calculated from NRLMSISE-00 is equal to the temperature of AO. The contribution to AO flux due to surface reflection is ignored.

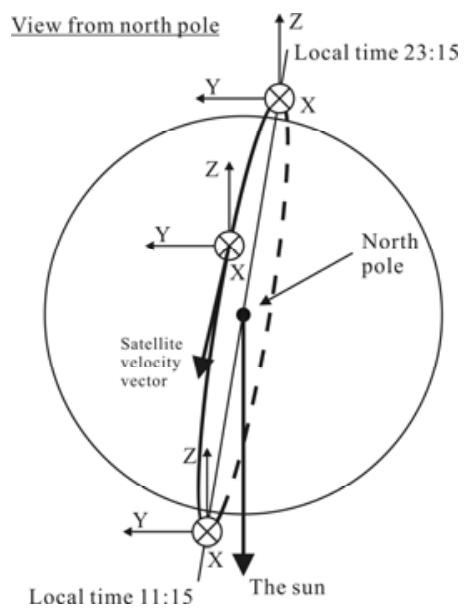

Fig. 6. The relationship between the satellite orbit and satellite coordinate system.

\section{Analysis Results and Discussion}

\subsection{Setting of input parameters}

In order to analyze AO flux distribution on the whole external surface of the ASNARO satellite, we need to know the predicted values of F10.7 flux and Ap index during the ASNARO mission.

For F10.7 flux, it is possible to use the data set predicted by the NASA Marshall Space Flight Center. ${ }^{9}$ Figure 7 shows the predicted value of F10.7 flux as of March 2009. We need to set the values of input parameters, that is, F10.7A and F10.7. F10.7A is the 81-day average of F10.7 flux and F10.7 is the daily F10.7 flux for the previous day. We use the expected 50 percentile level of predicted data for the input parameter F10.7A and the expected 95 percentile level of predicted data for the input parameter F10.7.' Figure 8 shows the relationship between F10.7 and F10.7A observed from 1947 to $2009 .{ }^{10)}$ The input parameters F10.7 and F10.7A used in this analysis are shown in Fig. 8. Note that the F10.7 input parameters used in this analysis do not include all past observation data.

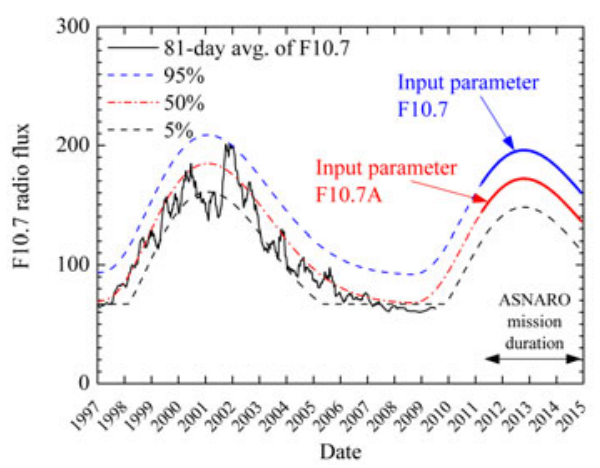

Fig. 7. Prediction of F10.7 flux. ${ }^{9}$

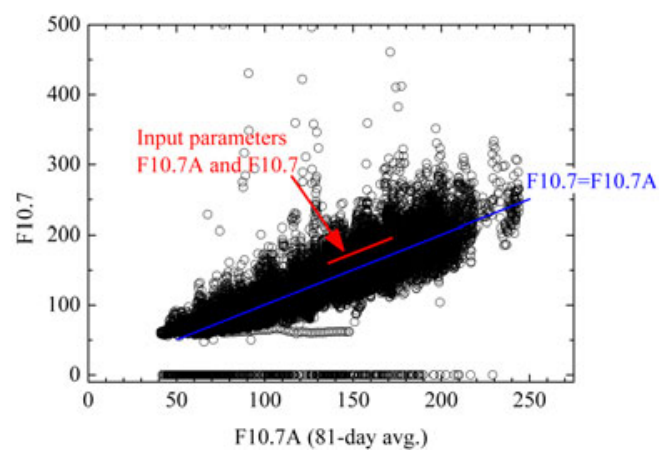

Fig. 8. Relationship between observation flux and input parameter.

For the Ap index, it was not easy to make a clear prediction. Therefore, it is difficult to set an adequate value of Ap for future satellite missions. Figure 9 shows the observed data of the Ap index from 1947 to 2009. In this study, we set the parameter Ap to 165 assuming the worst-case scenario. The observation data of the Ap index exceeding 165 is less than $0.1 \%$ during 1947 to $2009^{11)}$ as shown in Fig. 10.

Through use of the input parameters F10.7, F10.7A and 
Ap described above, the AO flux distribution is estimated for the ASNARO satellite mission during April 2011 to December 2014 based on the ASNARO being planned for launch in 2011, at the beginning of this AO fluence analysis project.

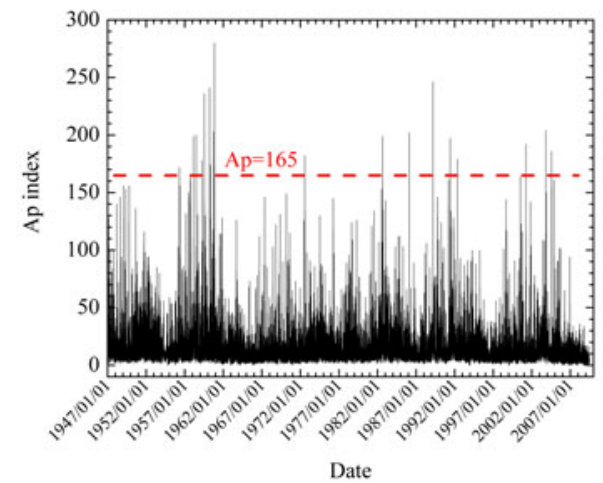

Fig. 9. Observed data of Ap index from 1947 to 2009. ${ }^{11)}$

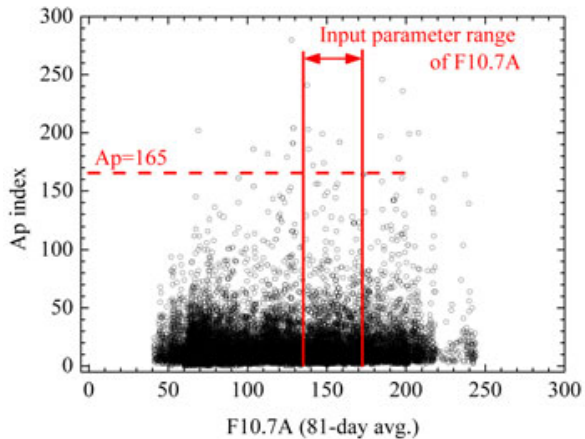

Fig. 10. Relationship between observed data of F10.7A and Ap index from 1947 to $2009 .^{10,11)}$

\subsection{AO flux distribution analysis}

We estimate the velocity vector, density and temperature of $\mathrm{AO}$ at intervals of 15 degrees in both latitude and longitude as the input parameters of MUSCAT.

As shown in Fig. 6, the ASNARO solar panels face in the direction of the sun, except during observation of the Earth's surface. Therefore, the translation velocity vector of rarefied AO gas flow is determined by the orbital position. The local time in the orbit is almost constant, as described above. The density and temperature of AO is determined using the parameters of local time, geodetic latitude and longitude of the orbit described in section 2 .

The MUSCAT analysis results are shown in Figs. 11 and 12 based on use of the input parameters velocity vector, density and temperature. The average AO flux during the ASNARO mission is shown. The analysis results in Figs. 11 and 12 are the amount of total AO fluence divided by the mission duration. If the analysis results of Figs. 11 and 12 are multiplied by the mission duration time, the total AO fluence during the ASNARO mission is obtained.

As shown in Figs. 12(a) and (b), the AO flux on the satellite surfaces facing the $\mathrm{X}$ plane is higher than on other surfaces. AO densities reach a maximum on the dayside on the equatorial plane as shown in Fig. 1. The satellite surfaces facing the $\mathrm{X}$ plane are exposed to high $\mathrm{AO}$ density atmosphere on ascending or descending nodes. Therefore, the satellite body surface and solar array panel edge facing the $\mathrm{X}$ plane receive high AO flux compared with the other surfaces, as shown in Fig. 13. The AO flux is lowest on the satellite body surfaces facing the $+Y$ plane as shown in Fig. 12(c) because the surfaces are on the wake-side surface during travel in the southern hemisphere, and the solar array panels obstruct the incidence of AO during travel in the northern hemisphere, as shown in Fig. 14. The maximum AO flux is $1.38 \times 10^{17}$ atoms $/ \mathrm{m}^{2} / \mathrm{s}$ on the whole surface of ASNARO.

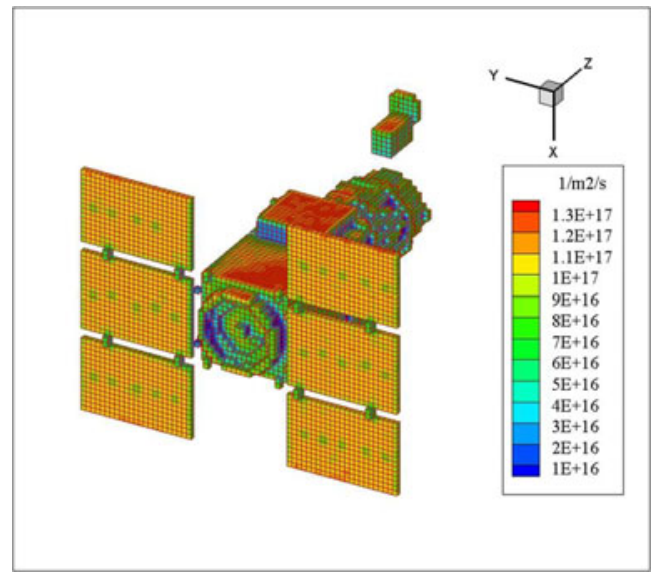

Fig. 11. Analysis results for the whole satellite surface.

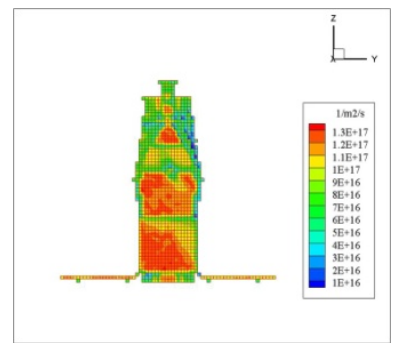

(a) $+\mathrm{X}$

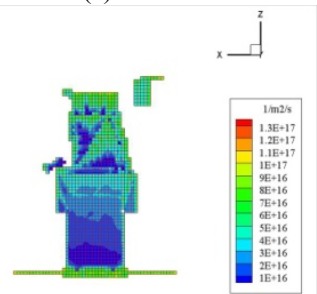

(c) $+\mathrm{Y}$

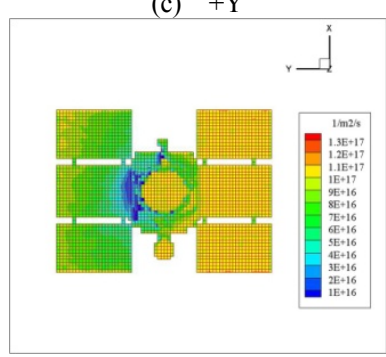

(e) $+Z$

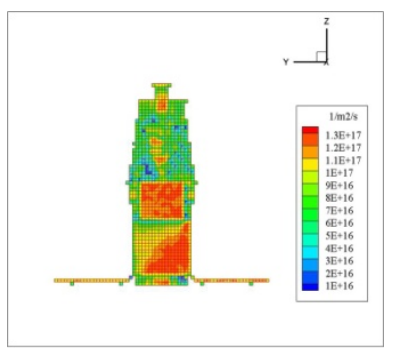

(b) $-\mathrm{X}$

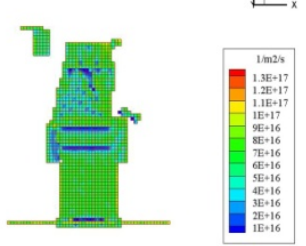

(d) $-\mathrm{Y}$

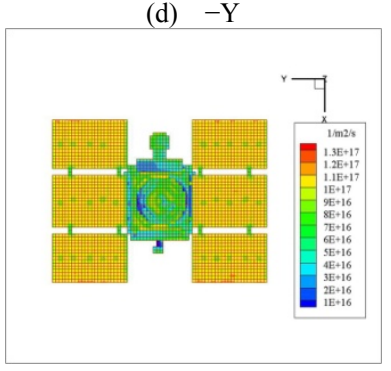

(f) $-\mathrm{Z}$
Fig. 12. AO flux distribution from the view of each axis. 


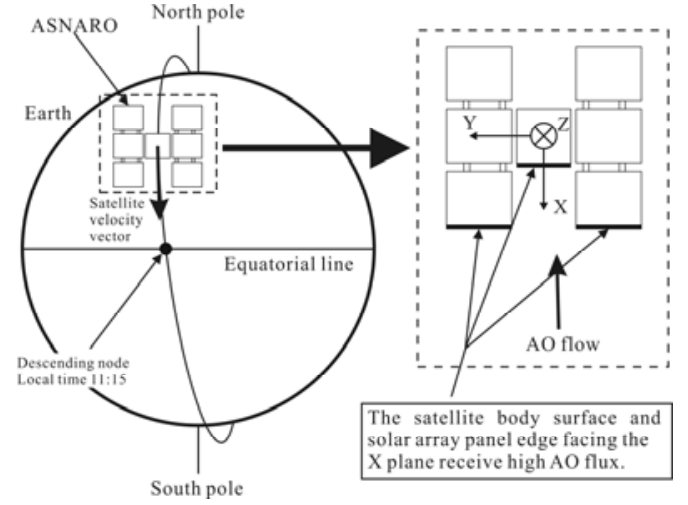

Fig. 13. Scheme of AO exposure on the satellite surfaces facing the $\mathrm{X}$ plane.

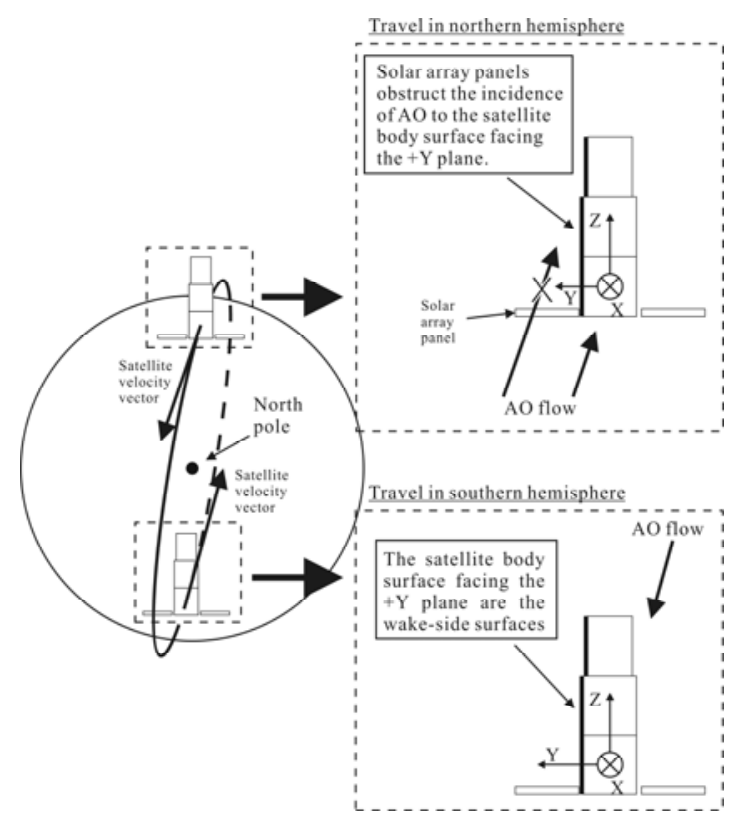

Fig. 14. Scheme of AO exposure on the satellite surfaces facing the $+Y$ plane.

\section{Future Plans}

The AO distribution analysis tool developed in this study could also be used for AO distribution analysis at exposure test facilities such as MPAC\&SEED ${ }^{4)}$ and MISSE. ${ }^{5)}$ Since the number of AO monitor materials installed in exposure test facilities is limited, it is impossible to identify the AO fluence of each exposed test sample by measuring the mass loss of an exclusive monitor material. The AO distribution analysis tool makes it possible to estimate the AO fluence of each exposed test sample by interpolating some fluence data from the AO monitor materials. By analyzing the AO fluence distribution at the beginning of the project in a space environment exposure test, it is also possible to determine the adequate position of the exposure test facility on spacecraft and $\mathrm{AO}$ monitor materials on a sample tray.

We plan to add a new function to the AO fluence analysis tool, namely, analysis of surface erosion distribution. The output includes the erosion rate, or reaction efficiency, due to AO depending on energy, incident angle and temperature. In addition, there is the synergistic effect with ultraviolet rays.
Therefore, we take into account the physical properties of reaction efficiency such as the energy, incident angle, temperature dependence and synergistic effect with ultraviolet rays. We subsequently analyze the AO erosion distribution in consideration of temperature and ultraviolet ray irradiance distribution on a spacecraft surface using the refined AO fluence analysis tool. It is necessary to verify the appropriateness of the model in which AO moves around behind the satellite wake-side surface with thermal velocity. In addition, the AO fluence analysis using the predicted data of Ap index and the adequate AO temperature is a topic for future studies.

\section{Summary}

In this study, we analyzed the distribution of AO flux on the whole surface of the ASNARO small Earth-observation satellite using the empirical neutral atmospheric model NRLMSISE-00 and MUSCAT. The analysis results showed that the AO flux on the satellite surface was non-uniform as a result of interference of the $3 \mathrm{D}$ satellite dimension and ram direction. The AO distribution analysis tool developed in this study was effective in evaluating the $\mathrm{AO}$ fluence environment of a satellite and exposure test facility in space.

\section{References}

1) Leger, L. J. and Visentine, J. T.: A Consideration of Atomic Oxygen Interactions with the Space Station, J. Spacecraft and Rockets, 23 (1986), pp.505-511.

2) Levine, A. S.: LDEF- 69 Months in Space Third Post-Retrieval Symposium, NASA-CP-3275, 1993.

3) Zimcik, D. G. and Maag, C. R.: Results of Apparent Atomic Oxygen Reactions with Spacecraft Materials During Shuttle Flight STS-41G, Journal of Spacecraft, 25 (1988), pp.162-168.

4) Kimoto, Y., Ichikawa, S., Miyazaki, E., Matsumoto, K., Ishizawa, J., Shimamura, H., Yamanaka, R. and Suzuki, M.: Space Environment Effects on Materials at Different Positions and Operational Period of ISS, Proceedings of the 9th International Conference on Protection of Materials and Structures from Space Environment, 2009, pp.207-211.

5) Dever, J. A., Miller, S. K., Sechkar, E. A. and Wittberg, T. N.: Space Environment Exposure of Polymer Films on the Materials International Space Station Experiment: Results from MISSE 1 and MISSE 2, High Performance Polymers, 20 (2008), pp.371-387.

6) Picone, J. M., Hedin, A. E., Drob, D. P. and Aikin, A. C.: NRLMSISE-00 Empirical Model of the Atmosphere: Statistical Comparisons and Scientific Issues, J. Geophys. Res, 107 (2002), pp.15-1-16.

7) Ogawa, T.: Development of the ASNARO, an Advanced Space System, NEC Technical Journal, 6 (2010), pp.36-40.

8) Muranaka, T., Hosoda, S., Kim, J., Hatta, S., Ikeda, K., Hamanaga, T., Cho, M., Usui, H., Ueda, H., Koga, K. and Goka, T.: Development of Multi-Utility Spacecraft Charging Analysis Tool (MUSCAT), IEEE Transactions on Plasma Science, 36 (2008), pp.2336-2349.

9) Solar cycle prediction http://solarscience.msfc.nasa.gov/predict.shtml

10) Solar radio data http://www.ngdc.noaa.gov/stp/solar/solarradio.html

11) Kp index data http://wdc.kugi.kyoto-u.ac.jp/kp/index-j.html 